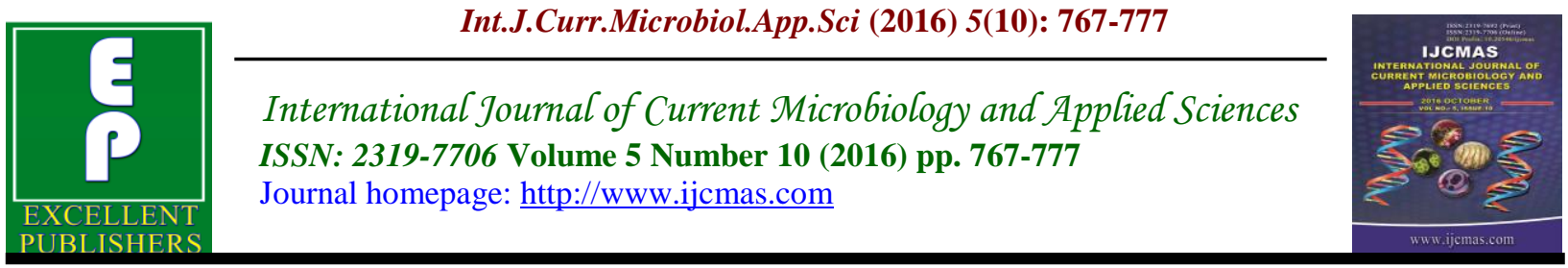

Original Research Article

http://dx.doi.org/10.20546/ijcmas.2016.510.083

\title{
Assessing the Appropriate Use of Proton Pump Inhibitors amongst the Outpatient Lebanese Population
}

\author{
George T. Nawas ${ }^{1}$, Tarek Nawas ${ }^{2}$, Diana Malaeb ${ }^{3}$, Sarah El Helou ${ }^{4}$ and Souheil Hallit ${ }^{4,5}$ \\ ${ }^{1}$ Lebanese American University, School of Pharmacy, Byblos, Lebanon \\ ${ }^{2}$ Lebanese American University, School of Arts and Sciences, Beirut, Lebanon \\ ${ }^{3}$ Lebanese International University, School of Pharmacy, Beirut, Lebanon \\ ${ }^{4}$ Lebanese University, School of Pharmacy, Hadath, Lebanon \\ ${ }^{5}$ Universite Saint-Esprit de Kaslik, School of medicine, Kaslik, Lebanon \\ *Corresponding author
}

Keywords

Proton Pump

Inhibitors,

Heartburn, Ulcer,

GERD, Lebanon,

Community

pharmacy.

\begin{tabular}{l}
\hline Article Info \\
\hline Accepted: \\
22 September 2016 \\
Available Online: \\
10 October 2016
\end{tabular}

A B S T R A C T

Proton pump inhibitors (PPI) are commonly prescribed for the treatment of many conditions but are currently being misused. The objective of this study was to evaluate the appropriate indication, dosage and duration of use of PPIs by outpatients in Lebanon. This cross-sectional study evaluated the treatment of 323 outpatients who presented to community pharmacies, in different Lebanese regions, with any PPI prescription. The results showed that there was a significant association between the prescribing of the PPI for the correct indication and recommending it in the appropriate dosage, but not with the type of PPI used nor with the duration of treatment that was indicated. No significant difference was found between the prescribed and over-the-counter PPIs regarding the appropriate duration of treatment, dosage and indication. A significant association was found, however, between the reason for PPI use and the other chronic diseases that the patients had and the number of cups of coffee drunk by a patient per day. PPI use by Lebanese outpatients showed to be mostly consistent with the FDA recommendations with regards to the dosage taken. However, the duration of therapy was clearly not in accordance with what is currently recommended. Physicians need to follow-up with patients and reassess the need for therapy on a regular basis.

\section{Introduction}

Proton pump inhibitors (PPIs) are one of the most commonly prescribed classes of medications worldwide both in the community and primary care settings. PPIs, the most potent class of drugs for the treatment of acid-related diseases, have replaced the histamine 2 receptor-antagonists
( $\mathrm{H}_{2} \mathrm{RA}$ 's) since the late 1980s (Wolfe, 2013). Evidence-based guidelines support PPI use, and are Food and Drug Administration(FDA) approved for the treatment and maintenance of healing erosive esophagitis (EE) and duodenal ulcers (DU), for the treatment of 
gastroesophageal reflux disease (GERD) and pathological hypersecretory conditions, including Zollinger-Ellison (ZE) syndrome, for the eradication of Helicobacter pylori in combination with antibiotics to reduce the risk of DU recurrences and for risk reduction of gastric ulcers (GU) that are associated with nonsteroidal antiinflammatory drugs (NSAIDs) (CMS, 2013).

Despite the fact that PPIs are considered to be safe medications, their overuse, misuse and extended use have been associated with significant adverse events including community-acquired pneumonia infections, spine, wrist and hip fractures, hypomagnesemia, and Clostridium difficile infections (Eurich et al., 2010; Yang, et al., 2006; Kaye and Jick, 2008; Florentin and Elisaf, 2012; Linsky et al., 2010; Dangler, 2013). The effectiveness of PPIs has led to their inappropriate utilization, exposing patients to an increased number of potential risks. Despite the US Food and Drug Administration's labeled indications and dosing (Lexicomp, 2011; Eisai Inc., 2013; Astra Zeneca, 2013a,b; TAP Pharmaceuticals Inc, 2013; Wyeth Pharmaceuticals Inc., 2013; Santarus Inc., 2013).

PPI therapy is still being prescribed for unapproved indications, in inappropriate doses and/or for longer term treatment. One of the major reasons for this overutilization is that the PPI therapy is initiated upon hospital admission to prevent stress ulcer, but therapy is not revaluated at discharge (Nardino et al., 2000). One study found that among patients prescribed acid suppressive therapy for ulcer prophylaxis during a hospital stay, $65 \%$ of patients did not have an indication for use, and 55\% were discharged on PPI therapy (Nasser et al., 2010).
Various studies that targeted the evaluation of PPI use were conducted worldwide, including studies from Ireland (Haroon et al., 2013; Walker and Mc Donald, 2001), Australia (Naunton, 2000), and Greece (Ntaios et al., 2009). However, published studies assessing the appropriate utilization of PPIs amongst the Lebanese population, and more specifically, at the community level are lacking. Hence, the objective of this study was to evaluate the PPI use in a sample of the outpatient Lebanese population and comparing it to the adopted international standards.

\section{Methods}

\section{Study design and sample size}

This cross-sectional study was conducted between the months of June and July 2015 on adult Lebanese patients recruited from different pharmacies across multiple Lebanese regions. A sample of 323patients was targeted to allow for adequate power for bivariate and multivariate analyses to be carried out according to the Epi info sample size calculations with a population size of 4 millions in Lebanon, a $30 \%$ expected frequency of PPI use and a 5\% confidence limits (CDC, 2016). Three hundred fifty questionnaires were distributed after taking the cluster effect and refusals into account.

\section{Inclusion criteria}

All patients above 18 years of age being already treated by PPI or presenting to the pharmacy and asking for a refill or are about to start a PPI course were considered for enrollment in the study. Patients with previous allergic reactions to PPIs were not eligible to participate. Verbal informed consent was obtained from all patients prior to participating in the study and completing the questionnaire. The Institutional Review 
Board of the Lebanese International University stated that approval was not necessary, since the study was an observational one and not experimental, clinical, or interventional.

\section{Data collection and measurement}

Data were collected using a structured questionnaire composed of 32 questions chosen and organized based on a thorough review of similar literature. The questionnaire was administered, in different pharmacies in the five main governorates of Lebanon, by trained interviewers in the Arabic language, the native language of the country, for ease of patient comprehension. The tool was pilot tested on 20 patients who were not included in the final study sample. For illiterate patients, the questionnaire was read aloud by the interviewers who marked the patient's answers on the paper. The questionnaire assessed socio-demographic characteristics, including age, gender, region and level of education and lifestyle characteristics, namely, smoking habits as to the kind of smoke (cigarettes and/or waterpipe smoking) and the number of cigarettes or water-pipes smoked per week; the consumption of alcohol (and how many glasses per day), drinking coffee and number of cups per day and finally the ingestion of spicy food and/or drinking acidic beverages (and their frequency per week). The core questions were, however, about the PPI they were coming to order, along with its dosage, frequency of administration, the duration of treatment and the reason for its prescription. The combination of PPIs in 2 groups, prescription versus over-the-counter PPI that we followed was the same as the categories followed in the United States of America (TAP Pharmaceuticals Inc., 2013; AstraZeneca, 2013b). Accepted indications of the PPI were those that were approved by the United States Food and Drug Administration or strongly supported by the Education Medicaid Integrity Contractor (MIC) for the CMS Medicaid Integrity Program (MIP) ${ }^{2}$, namely: treatment of active ulcer disease; maintenance in high-risk ulcer patients who did not have evidence of Helicobacter pylori infection; erosive esophagitis or gastritis; symptomatic gastric esophageal reflux; "stress" ulcer prophylaxis in high-risk patients (defined as patients with coagulopathy or prolonged mechanical ventilation); and use of proton pump inhibitors as part of a treatment regimen for H. pylori. Certain other uses were believed to be acceptable, including: relief of dyspepsia caused by nonsteroidal antiinflammatory drugs (NSAIDs), prevention of NSAID-induced ulcers (proton pump inhibitors only) and trials of therapy for nonulcer dyspepsia. Any other use was considered a misuse of the PPI treatment. The dosage and duration of the PPI use were also assessed according to each indication of use as cited in the MIC reference (CMS, 2013).

\section{Statistical analysis}

Statistical analysis was performed using SPSS software version 21. Responses to questions were coded and entered into a database for descriptive and comparative analysis. Descriptive statistics mainly mean values and standard deviation (SD) were presented for continuous quantitative variables, while frequencies and percentages were used for nominal and ordinal variables. Student's $t$-tests were conducted to examine differences in quantitative variables while Chi-square analyses were used to compare qualitative and some categorical variables. A $p$-value less than 0.05 was considered to be statistically significant. It is important to mention that not all totals sum to the sample size because of missing data from some 
questions that were not answered by the patients.

\section{Results and Discussion}

\section{Socio-demographic and lifestyle characteristics}

Out of the 350 distributed questionnaires, $323(92.3 \%)$ were filled. Table 1 summarizes the socio-demographic characteristics of the patients surveyed. The sample consisted of $163(50.5 \%)$ males and $160(49.5 \%)$ females. The mean age of the total sample was $41.58 \pm 1.84$ years with no difference between males and females. The biggest clusters of the patients lived in Mount Lebanon (48.3\%), and in Beirut $(35.3 \%)$.Half $(50.1 \%)$ of the survey participants held a university degree, with a higher percentage among males (55.6\%) compared to females (45.2\%).

Table 2, which summarizes the lifestyle habits of the patients, shows that of the patients taking part in the study, $42.7 \%$ were current smokers, with a higher percentage of males $(56.4 \%)$ compared to females $(28.1 \%)$ and an average of $12 \pm 10.48$ cigarettes smoked per day for the total sample, noting that there was a significant difference in cigarette smoking between genders, higher in males versus females $(\mathrm{p}<0.001)$; however, there was no gender differential for waterpipe smoking. Of their other daily habits, $50.8 \%$ of the patients consumed alcohol with an average of $2.69 \pm 1.15$ glasses per week. The patients who drank coffee were $75.8 \%$ of the participants, with an average of $4.4 \pm 0.98$ cups per day. On the other hand, $60.1 \%$ ate spicy food at an average of $2.45 \pm$ 1.22 times per week, while $67.2 \%$ consumed acidic beverages with an average of $3.64 \pm$ 2.85 times per week.

The ANOVA test revealed a significant difference between the number of cups of coffee drunk per day and the reason for taking PPI versus those who did not drink coffee $(F=2.291 ; p=0.036)$. To note that the association between the reason for taking PPI and the quantity of spicy food eaten per week tended towards significance when compared with those who did not have any spicy food $(\mathrm{p}=0.052)$.

\section{Observations concerning the use of PPIs}

The most commonly used PPI drug was omeprazole $(42.9 \%)$ followed by esomeprazole $(23.1 \%)$, rabeprazole $(20.1 \%)$, pantoprazole $(6.5 \%)$ and lansoprazole $(6.5 \%)$. Table 3 summarizes facts about the use of PPI drugs in our sample of the Lebanese community. The biggest portion of the patients (34.4\%) took one of the PPIs for stomach protection, the latter being the fear of having stomach problems resulting from polypharmacy without having any history of gastro-intestinal problems. In addition, $23.8 \%$ used them for GERD problems $19.5 \%$ as a concomitant treatment with NSAIDs and $14.9 \%$ for gastric ulcer.

The association between the reason for taking a PPI and the PPI drug used, along with the appropriate dosage and duration of treatment is shown in Table 3. There was a significant association between the appropriate use of PPI and the appropriate dosage $(p=0.038)$. However, the duration of treatment as well as the active ingredient used were not significantly correlated with the appropriate indication for use.

Table 4 shows that $50.2 \%$ of the consumed PPIs were based on a doctor's prescription. Of these, $33.3 \%$ of the patients had a treatment duration of 6 months or more while $9.3 \%$ took it for one week only. Only about half of the patients $(50.3 \%)$ took their PPI for the appropriate indication, with only $24.8 \%$ taking it for the appropriate duration of treatment and $34.8 \%$ of them taking the 
appropriate dosage. The use of PPI over the counter (OTC) (omeprazole, lansoprazole) to the prescribed ones (rabeprazole, esomeprazole, pantoprazole) was also compared (Table 4) with regards to the appropriate indication, duration of treatment and dosage. No significant association was found between these 2 groups concerning all parameters tested ( $p>0.05$ for all).

Table 5 shows that there was no significant difference between doctor's prescribing the PPI and the appropriate indication, duration of treatment or strength of the PPI ( $p>0.05$ for all variables).

In this study, there was no significant difference between the use of PPI and gender $(p=0.676)$. However, there was a significant association between the reason for PPI use and the comorbidities that the patient might have had (cardiac, diabetes, hypertension, dyslipidemia) $(\mathrm{p}<0.001)$.

On the other hand, there was no significant difference between the reason for PPI intake and the number of cigarettes/water-pipes smoked per day $(p=0.476)$, the type of smoking (cigarettes/water-pipe) ( $)$ $0.644)$, the number of alcohol glasses per week $(p=0.318)$, the kind of alcohol drunk $(\mathrm{p}=0.887)$, drinking acidic beverages $(p=0.110)$ or the number of acidic beverages drunk per week $(p=0.630)$ versus those who did not include each one of the above as part of their life habit, when compared to those who did not drink acidic beverages.

In this study of 323 adult Lebanese patients using PPIs, the use of PPIs in the community was investigated and the appropriateness as well as the misuse of these agents, which exposes patients to potential risks, were evaluated.

Inappropriate prescriptions of PPI have been widely reported, with $40-80 \%$ of all PPI prescriptions not being in agreement with standard guidelines (Mat Saad et al., 2005; Strid et al., 2003; Zink et al., 2005; Boutet et al., 1999).

This study highlights the common practice of overuse of PPIs since they are very commonly used for stomach protection but are in appropriately prescribed or are prescribed for an unsuitable duration of therapy. Only half of our study participants took their PPI for the appropriate indication, with solely $25.9 \%$ who took it for the appropriate duration of treatment. In addition, only $34.6 \%$ of the patients took their PPIs in the appropriate dosage. Previous research on potential PPI overuse in managed care settings reported an estimated $6 \%$ to $24 \%$ incidence of patients taking AST without an appropriately documented indication (Heidelbaugh, 2010).

According to the results of this study, there was a significant association between the reason of PPI use and the other diseases that the patient might have had. Conversely, a study done in Michigan showed no statistical association between PPI use and comorbid conditions to demonstrate any potential cause-and-effect relationship, taking into consideration that the most common comorbid conditions were related to cardiovascular disorders, including hypertension and hyperlipidemia (65\%), whereas the least common comorbidities were related to orthopedic conditions $(0.1 \%)$ (Krstić et al., 2013).

The study also demonstrated a significant association between the daily coffee consumption and the reason for taking PPI. However, the number of cigarettes/ waterpipes smoked per day, the type of smoking (cigarettes/ water-pipe), the number of alcohol glasses per week and the kind of alcohol consumed were not significantly associated with the reason of PPI uptake. 
These findings were not compatible with a Serbian study in which coffee consumption $(86.1 \%)$ was the most common life habit to patients suffering from GERD (and thus requiring PPI therapy), followed by exposure to stress (81.7\%), smoking (45.7\%) and alcohol consumption (25\%), noting that with PPI therapy a statistically significant reduction of typical GERD symptoms of any intensity was detected as well as the reduction in the frequency of all symptoms and accompanying discomfort (Katz et al., 2013).

Furthermore, current consensus guidelines suggest lifestyle modifications including weight loss and head of bed positioning as more effective strategies to manage symptoms of GERD than limiting highly acidic or spicy foods (Lødrup et al., 2014). Indeed, our results revealed that drinking acidic beverages or the number of acidic beverages drunk per week didn't have any significant association with PPI intake, whereas the association between the reason for taking PPI and the quantity of spicy food eaten per week tended to significance since many people use PPI for the relief of the episodes of heartburn caused by spicy food consumption.

In comparing PPI usage between genders, this study did not demonstrate a significant difference. In contrast, a study performed in Denmark, showed that a female gender significantly increased the risk of PPI use among other factors such as old age, use of non steroidal anti-inflammatory drugs (NSAIDS) or anti platelet therapy (Lødrup et al., 2014).

Moreover, this study revealed that the most popular PPI used by the Lebanese patients was omeprazole, used by $42.9 \%$ of the participants. Our findings didn't match those of an Irish study that showed esomeprazole as the most commonly used PPI (38\%) followed by pantoprazole (34\%) whereas omeprazole was used only by $11 \%$ of the participants ${ }^{18}$ in that study. It is important, however, to mention that in Lebanon, all medications, except for controlled substances, can be dispensed with or without a physician's prescription.

\section{Limitations}

To our knowledge, this is the first study to analyze the appropriateness of PPI treatment recommendations in Lebanese ambulatory patients and to identify factors associated with the continuation of appropriately and inappropriately prescribed PPI. Although a representative sample of patients based on demographics and regional distribution was included in the study, yet, the total sample size is considered small and might not be representative of the whole Lebanese population. In addition, some of our results may not be purely indicative of the characteristics of all Lebanese patients taking PPI since most of the participants were from two main governorates of Lebanon, Mount Lebanon and Beirut. Hence any generalization is limited and selection bias could not be excluded. In addition, although the interviewers filling up the questionnaires were well trained, yet, it is possible that they failed to ask few of the questions. This has potential disadvantages concerning information bias and eliciting only social acceptable responses, despite the fact that we ensured anonymity and confidentiality of all data that has been collected. Moreover, our study did record the incidence of reported adverse events with a potential relationship to PPI therapy and didn't examine the suspected effects of PPI therapy on anti platelet agents. 
Table.1

Table 1. The socio-demographic characteristics of the participants in the study

Factor

- Age (in years)

- Gender

Male

Female

$\frac{\text { Mean } \pm \text { SD }}{41.58 \pm 1.84} \quad \underline{\mathrm{N}(\%)}$

- Level of education

Illiterate

$<8$ years of studies

$>8$ years of studies

University

Not available

\section{- Governorate}

Beirut

$114(35.3 \%)$

Mount Lebanon

North

South

Bekaa
$163(50.5 \%)$

$160(49.5 \%)$
$27(8.4 \%)$

$55(17 \%)$

$76(23.5 \%)$

$162(50.1 \%)$

$3(1 \%)$

Table.2

Table 2. The lifestyle characteristics of the participants in the study

Factor

$\underline{\text { Mean } \pm \mathrm{SD}} \quad \underline{\mathrm{N}(\%)}$

- Smoker

No

$185(57.3 \%)$

Yes

- Smoking kind

Cigarette

79 (24.4\%)

Water-pipe

$29(9 \%)$

Both

$30(9.3 \%)$

Mean number of cigarettes per week

$12 \pm 10.48$

- Alcohol intake 
No

Yes

Not available

Mean glasses per week

\section{- Coffee intake}

No

Yes

Not available

Mean cups per day
$158(48.9 \%)$

$164(50.8 \%)$

$1(0.3 \%)$

\section{- Spicy food consumer}

No

$194(60.1 \%)$

Yes

$128(39.6 \%)$

$1(0.3 \%)$

Not available

$2.45 \pm 1.22$

Mean times consumed per week

$$
\begin{array}{r}
72(22.3 \%) \\
245(75.8 \%) \\
6(1.9 \%)
\end{array}
$$

$4.4 \pm 0.98$

\section{- Acidic beverages}

No

$106(32.8 \%)$

Yes

Mean times consumed per week

$3.64 \pm 2.85$

Table.3 Association between the appropriate use of the PPIs and their indications

$\begin{array}{lcccccc}\begin{array}{l}\text { Reason for } \\ \text { PPI/Factor }\end{array} & \begin{array}{c}\text { Stomach } \\ \text { protection }\end{array} & \begin{array}{c}\text { Along with } \\ \text { NSAID } \\ \text { treatment }\end{array} & \text { Ulcer } & \text { GERD } & \begin{array}{c}\text { Other } \\ \text { reasons }\end{array} & \begin{array}{c}\text { p- } \\ \text { value }\end{array} \\ \begin{array}{l}\text { Total } \\ \text { Appropriate duration } \\ \text { of treatment }\end{array} & \mathbf{1 1 1}(\mathbf{3 4 . 4 \% )} & \mathbf{6 3}(\mathbf{1 9 . 5 \% )} & \mathbf{4 8 ( 1 4 . 9 \% )} & \mathbf{7 7}(\mathbf{2 3 . 8 \% )} & \mathbf{2 4}(\mathbf{7 . 4 \% )} & \text { - } \\ \text { No } & 87(78.4 \%) & 46(73 \%) & 33(68.8 \%) & 56(72.7 \%) & 16(66.7 \%) & \\ \text { Yes } & 24(21.6 \%) & 17(27 \%) & 15(31.2 \%) & 21(27.3 \%) & 8(33.3 \%) & \\ \text { Appropriate dosage } & & & & & & 0.64 \\ \text { No } & 77(69.4 \%) & 47(74.6 \%) & 23(47.9 \%) & 49(63.6 \%) & 14(58.3 \%) & \\ \text { Yes } & 34(30.6 \%) & 16(25.4 \%) & 25(52.1 \%) & 28(36.4 \%) & 10(41.7 \%) & \\ \text { PPI kind } & & & & & & \\ \text { Omeprazole } & 53(48.2 \%) & 24(38.1 \%) & 22(46.8 \%) & 32(42.1 \%) & 8(33.3 \%) & \\ \text { Esomeprazole } & 23(20.9 \%) & 16(25.4 \%) & 7(14.9 \%) & 19(25 \%) & 10(41.7 \%) & \\ \text { All others } & 34(30.9 \%) & 23(36.5 \%) & 18(38.3 \%) & 25(32.9 \%) & 6(25 \%) & \\ & & & & & \end{array}$


Table.4 Comparison between OTC and Rx PPI use.

\begin{tabular}{|c|c|c|c|c|}
\hline PPI categories/Factors & Total & $\begin{array}{l}\text { OTC PPI } \\
\text { N=160 } \\
(\mathbf{4 9 . 8 \%})\end{array}$ & $\begin{array}{l}\text { Rx PPI } \\
\mathbf{N}=161 \\
(50.2 \%)\end{array}$ & P-value \\
\hline \multicolumn{4}{|l|}{ Appropriate indication } & \multirow[t]{3}{*}{0.956} \\
\hline No & $160(49.8 \%)$ & $80(50 \%)$ & $80(49.7 \%)$ & \\
\hline Yes & $161(50.2 \%)$ & $80(50 \%)$ & $81(50.3 \%)$ & \\
\hline \multicolumn{4}{|c|}{ Appropriate duration of treatment } & \multirow[t]{3}{*}{0.678} \\
\hline No & $238(74.1 \%)$ & $117(73.1 \%)$ & $121(75.2 \%)$ & \\
\hline Yes & $83(25.9 \%)$ & $43(26.9 \%)$ & $40(24.8 \%)$ & \\
\hline \multicolumn{4}{|l|}{ Appropriate dosage } & \multirow[t]{3}{*}{0.939} \\
\hline No & $210(65.4 \%)$ & $105(65.6 \%)$ & $105(65.2 \%)$ & \\
\hline Yes & $111(34.6 \%)$ & $55(34.4 \%)$ & $56(34.8 \%)$ & \\
\hline
\end{tabular}

Table.5 Comparison between the correct use of PPI and physicians' recommendations

Factor/Doctor's recommended

Appropriate indication

No

Yes

Appropriate duration of treatment

No

Yes

Appropriate dosage

No

Yes
Yes

$119(50.6 \%)$

$116(49.4 \%)$

$177(75.3 \%)$

$58(24.7 \%)$

$159(67.7 \%)$

$76(32.3 \%)$
No

$42(48.8 \%)$

$44(51.2 \%)$

Therefore, we suggest that further research be conducted taking into account these limitations; we also suggest qualitative research that can explore the knowledge, attitudes and values behind these behaviors.

\section{Significance}

This study suggests that PPI prescription in the Lebanese community setting may have areas for improvement. Adequate follow-up after initiation of therapy is a principle area of focus. After initial therapy prescription, prescribers are not reassessing patient symptoms within the FDA recommended guidelines for therapy duration and appropriate indication. So, patients continue the PPI drugs which they were originally started on by another provider and assume that therapy appropriateness was determined by the previous provider and, therefore, continue PPI therapy inappropriately.

We conclude that PPIs are often overused in the Lebanese community setting without documented valid indication for treatment or for continuation of therapy. Therefore, indication for PPI prescription should be assessed periodically. Physicians should carefully assess hospital discharge prescriptions as well as medication prior to admission, focusing on the indication for continuous prescription of PPIs to avoid under- and overprescribing of PPIs. Reasons 
for inadequate PPI prescriptions need to be explored in more depth to tailor interventions and educational programs for healthcare professionals promoting appropriate PPI prescribing.

Additional research to prove a causal relationship between PPI therapy and potential adverse events documented in the literature needs to be performed, as there is a paucity of data regarding this issue in the Lebanese community.

\section{Acknowledgment}

We thank the interviewers who worked hard in the data collection and Miss Nelly Kheir who assisted us in the data entry.

\section{References}

Astra Zeneca, L.P. 2013a. Nexium prescribing information [online], Available at: http://www1.astrazenecaus.com/pi/Nexium.pdf .

Astra Zeneca, L.P. 2013b. Prilosec prescribing information [online] Available at: http://www1.astrazenecaus.com/pi/Prilosec.pdf.

Boutet, R., Wilcock, M. and MacKenzie, I. 1999. Survey on repeat prescribing for acid suppression medications in primary care in Cornwall and the Isles of Sicily. Aliment Pharmacol. Ther., 13(6): 813817.

Centers for disease control and prevention (CDC). 2016. What is EpiInfo7. Available from: http://www.cdc.gov/epiinfo/7/index.htm 1

Dangler, M., Ochs, L. and White, R. 2013. Assessing the Appropriate Use of Proton Pump Inhibitors in a Veteran Outpatient Population. Fed. Pract., 2125.

Eisai Inc. 2013. Aciphex prescribing information [online] [Accessed March 11, 2013]. Available at: http://dailymed.nlm.nih.gov/dailymed/ar chives/fdaDrugInfo.cfm? archiveid=101 84.

Eurich, D.T., Sadowski, C.A., Simpson, S.H., Marrie, T.J. and Majumdar, S.R. 2010. Recurrent community-acquired pneumonia in patients starting acidsuppressing drugs. Am. J. Med., 123(1): 47-53.

Florentin, M. and Elisaf, M. 2012. Proton pump inhibitor-induced hypomagnesemia: A new challenge. World J. Nephrol., 1(6): 151-154. DOI: 10.5527/wjn.v1.i6.151

Haroon, M., Yasin, F., Gardezi, K.M., Adeeb, F. and Walker, F. 2013. Inappropriate use of proton pump inhibitors among medical inpatients: a questionnairebased observational study, JRSM Short Rep., 4(8).

Heidelbaugh, J.J., Goldberg, K.L. andInadomi, J.M. 2010. Magnitude and Economic Effect of Overuse of Antisecretory Therapy in the Ambulatory Care Setting. Am. J. Manag. Care, 16(8): e228-234.

Katz, P.O., Gerson, L.B. and Vela, M.F. 2013. Guidelines for the diagnosis and management of gastroesophageal reflux disease. Am. J. Gastroenterol., 108 (3): 308-328; DOI: 10.1038/ajg.2012.444.

Kaye, J.A. and Jick, H. 2008. Proton pump inhibitor use and risk of hip fractures in patients without major risk factors. Pharmacother., 28(8): 951-959.

Krstić, M., Damjanov, D. and Ngorni, A. 2013. Observational study in primary health care: symptoms control of gastroesophageal reflux disease and influence on the quality of life. Srp Arh Celok Lek., 141(1-2): 54-60.

Lexicomp (Online). Omeprazole Drug Information [Accessed June 2011]. Available at: www.UpToDate.com.

Linsky, A., Gupta, K., Lawler, E.V., Fonda, J.R. and Hermos, J.A. 2010. Proton pump inhibitors and risk for recurrent Clostridium difficile infection. Arch. Intern. Med., 170(9): 772-778. 
Lødrup, A., Pottegård, A., Hallas, J.andBytzer, P. 2014. Use of proton pump inhibitors after antireflux surgery: a nationwide register-based follow-up study. Gut., 63(10): 1544-1549. DOI: 10.1136/gutjnl-2

Mat Saad, A.Z., Collins, N., Lobo, M.M. and O'Connor, H.J. 2005. Proton pump inhibitors: a survey of prescribing in an Irish general hospital. Int. J. Clin. Pract., 59: 31-34.

Nardino, R.J., Vender, R.J. and Herber, P.N. 2000. Overuse of acid-suppressive therapy in hospitalized patients. Am. J. Gastroenterol., 95(11): 3118-3122.

Nasser, S.C., Nassif, J.G., and Dimassi, H.I. 2010. Clinical and cost impact of intravenous proton pump inhibitor use in non-ICU patients. World $J$. Gastroenterol., 16(8): 982-986. doi: 10.3748/wjg.v16.i8.982

Naunton, M., Peterson, G.M. and Bleasel, M.D., 2000. Overuse of proton pump inhibitors. J. Clin. Pharm. Ther., 25: 333-340.

Ntaios, G., Chatzinikolaou, A., Kaiafa, G., Savopoulos, C., Hazitolios, A. and Karamitsos, D., 2009. Evaluation of use of proton pump inhibitors in Greece. Eur. J. Intern. Med., 20(2): 171-173.

Santarus Inc. Zegerid prescribing information [online] [Accessed March 11, 2013]. Available at:http://www.zegerid.com/docs/zegerid -full-prescribing-information.pdf.

Strid, H., Simren, M. and Bjornsson, E.S. 2003. Overuse of acid suppressant drugs in patients with chronic renal failure. Nephrol. Dial. Transplant, 18: 570-575.

TAP Pharmaceuticals Inc. Prevacid prescribing information [online] [Accessed March 11, 2013]. Available at:

http://www.accessdata.fda.gov/drugsatf da_docs/label/2008/020406s067,021281 s024,021428s017lbl.pdf .

The Centers for Medicare \& Medicaid Services (CMS). 2013. Proton Pump Inhibitors: Use in Adults. Available from https://www.cms.gov/MedicareMedicaid-Coordination/FraudPrevention/Medicaid-IntegrityEducation/Pharmacy-EducationMaterials/Downloads/ppi-adultfactsheet.pdf

Walker, N.M. and McDonald, J. 2001. An evaluation of the use of proton pump inhibitors. Pharm. World Sci., 23: 116117.

Wolfe, M. 2013. Overview and comparison of proton pump inhibitors for the treatment of acid-related disorders. In: Basow DW, ed. UpToDate. Waltham, MA.

Wyeth Pharmaceuticals Inc. Protonix prescribing information [online] [Accessed March 11, 2013]. Available at:

http://labeling.pfizer.com/showlabeling. aspx?id=135.

Yang, Y.X., Lewis, J.D., Epstein, S. and Metz, D.C. 2006. Long term proton pump inhibitor therapy and risk of hip fracture. JAMA, 296(24): 2947-2953.

Zink, D.A., Pohlman, M., Barnes, M., and Cannon, M.E. 2005. Long-term use of acid suppression started inappropriately during hospitalization. Aliment Pharmacol. Ther., 21: 1203-1209. DOI: $10.1111 / \mathrm{j} .1365-2036.2005 .02454$.

\section{How to cite this article:}

George T. Nawas, Tarek Nawas, Diana Malaeb, Sarah El Helou and Souheil Hallit. 2016. Assessing the Appropriate Use of Proton Pump Inhibitors amongst the Outpatient Lebanese Population. Int.J.Curr.Microbiol.App.Sci. 5(10): 767-777. doi: http://dx.doi.org/10.20546/ijcmas.2016.510.083 